\title{
Transfusionsassoziierte akute Lungeninsuffizienz (TRALI)
}

\author{
Transfusion-related Acute Lung Injury (TRALI)
}

Autoren

Institute
H. Schweisfurth ${ }^{1}$, I. Sopivnik ${ }^{2}$, R. Moog ${ }^{2}$

1 Pulmologisches Forschungsinstitut (IPR)

${ }^{2}$ DRK Blutspendedienst Nord-Ost gGmbH, Institut Cottbus eingereicht 12.3.2014 akzeptiert nach Revision 28.5.2014

\section{Bibliografie}

Dol http://dx.doi.org/ 10.1055/s-0034-1377333

Online-Publikation: 21.7.2014

Pneumologie 2014; 68: 599-603

(c) Georg Thieme Verlag KG

Stuttgart · New York

ISSN 0934-8387

Korrespondenzadresse

Prof. Dr. med.

Hans Schweisfurth

Pulmologisches

Forschungsinstitut (IPR)

Walther-Rathenau-Straße 11

03044 Cottbus

ipr@pulmologisches-

forschungsinstitut.de

\section{Zusammenfassung \\ $\nabla$}

Die transfusionsassoziierte akute Lungeninsuffizienz (TRALI) wird hauptsächlich durch Transfusionen von frisch gefrorenem Plasma oder Thrombozytenkonzentraten verursacht und tritt definitionsgemäß innerhalb von 6 Stunden nach Transfusion mit akuter Atemnot, Hypoxämie und röntgenologisch nachweisbaren bilateralen Thoraxinfiltraten auf. Ursache sind meistens leukozytäre Antikörper im Plasma des Blutspenders (immunogene TRALI). Aber auch antikörperunabhängige Substanzen wie biologisch aktive Lipide, die vorwiegend bei der Lagerung von Thrombozyten- und Erythrozytenkonzentraten entstehen, können neutrophile Granulozyten aktivieren und eine nichtimmunogene TRALI auslösen. Pathophysiologisch setzen die Granulozyten in den Kapillaren der Lungenstrombahn Sauerstoffradikale und Enzyme frei, die die Endothelzellen schädigen und zum Lungenödem führen. Therapeutisch kann nasale Sauerstoffgabe ausreichen. In schweren Fällen sind mechanische Beatmung, invasive hämodynamische Überwachung und Flüssigkeitszufuhr erforderlich. Diuretika sind zu vermeiden. Die Gabe von Glukokortikoiden ist umstritten. Da antikörperbedingte TRALI-Reaktionen hauptsächlich nach Transfusion von frisch gefrorenem Plasma auftraten, das von Frauen gewonnen wurde, die sich im Verlauf von Schwangerschaften gegen leukozytäre Antigene immunisiert haben, wird in Deutschland seit 2009 nur Plasma von Spenderinnen ohne Schwangerschaften in der Anamnese oder nach negativer Testung auf Antikörper gegen HLA-Klasse I, II und HNA eingesetzt mit der Folge, dass seitdem kein TRALI-bedingter Todesfall mehr registriert wurde.

\section{Abstract \\ $\nabla$}

Transfusion-related acute lung injury (TRALI) is primarily caused by transfusion of fresh frozen plasma or platelet concentrates and occurs by definition within 6 hours after transfusion with acute shortness of breath, hypoxemia and radiographically detectable bilateral infiltrates of the lung. Mostly leucocyte antibodies in the plasma of the blood donor (immunogenic TRALI) are responsible. Apart from antibodies, other substances such as biologically active lipids, mainly arising from the storage of platelet and red blood cell concentrates, can activate neutrophilic granulocytes and trigger a non-immunogenic TRALI. Pathophysiologically, granulocytes in the capillaries of the lung vessels release oxygen radicals and enzymes which damage the endothelial cells and cause pulmonary edema. Therapeutically, nasal oxygen administration may be sufficient. In severe cases, mechanical ventilation, invasive hemodynamic monitoring and fluid intake are required. Diuretics should be avoided. The administration of glucocorticoids is controversial. Antibodyrelated TRALI reactions occurred mainly after transfusion of fresh frozen plasma, which had been obtained from womenimmunized during pregnancy against leukocyte antigens. Therefore, in Germany, since 2009 only plasma from female donors without a history of prior or current pregnancy or negative testing for antibodies against HLA I, II or HNA has been used with the result that since then no TRALI-related death has been registered. 


\section{Einleitung}

$\nabla$

Die transfusionsassoziierte akute Lungeninsuffizienz (TRALI) ist eine selten auftretende, oft lebensbedrohliche Transfusionsreaktion, die zumeist durch Transfusion von frisch gefrorenem Plasma oder Thrombozytenkonzentraten auftritt und durch irreguläre leukozytäre Antikörper im Spenderblut ausgelöst wird. Diese Transfusionsnebenwirkung wurde zunächst bei Erwachsenen durch Lanman 1950 [1] und Barnard 1951 [2] beschrieben und 1985 als Transfusion-related Acute Lung Injury bezeichnet [3]. TRALI ist inzwischen anerkannt als eine seltene, aber wichtige Komplikation von Transfusionen in allen Altersgruppen [4-8]. Obwohl die meisten Fälle von TRALI erfolgreich behandelt werden können, ist sie zurzeit eine der führenden Ursachen der Sterblichkeit nach Transfusion von Blutprodukten insbesondere von frischem gefrorenem Plasma [9-11]. Trotz ihrer Bedeutung wird TRALI vermutlich größtenteils nicht erkannt [12].

In den USA und Großbritannien ist TRALI die führende transfusionsassoziierte Todesursache [9]. Nach einem Bericht der britischen Melde- und Auswertungsstelle für Nebenwirkungen von Bluttransfusionen, dem SHOT („serious hazards of transfusion“) Report, war TRALI in Großbritannien inzwischen die häufigste Ursache für eine gravierende Transfusionsreaktion [13]. Durch die Bevorzugung von männlichen Spendern konnte TRALI erheblich reduziert werden $[14,15]$.

In Deutschland wurden im Januar 2009 durch den Arbeitskreis Blut des Bundesministeriums für Gesundheit Maßnahmen zur Vermeidung der TRALI beschlossen und im September 2009 durch das Paul-Ehrlich-Institut ein Stufenplan zur Umsetzung festgelegt [16]. Demnach darf in Deutschland das Plasma von weiblichen Spendern ohne Schwangerschaften in der Anamnese oder nach negativer Testung auf Antikörper gegen HLA-Klasse I, II und HNA zur Herstellung von therapeutischem Plasma verwendet werden.

\section{Definition \\ $\nabla$}

Das Europäische Hämovigilanz-Netzwerk (EHN) definierte TRALI als die klinische Entität bestehend aus akuter Atemnot während oder innerhalb von 6 Stunden nach Transfusion von Blutprodukten, verbunden mit neu aufgetretenen bilateralen röntgenologisch nachweisbaren Lungeninfiltrationen (Lungenödem) ohne Anhalt für eine Herzinsuffizienz infolge Volumenüberladung [17].

Das nordamerikanische TRALI-Konsensus-Konferenz-Komitee hat den Nachweis der Hypoxämie sowie den Ausschluss weiterer Risikofaktoren für die Auslösung eines akuten Lungenversagens („acute lung injury“, ALI) wie Sepsis, Schock, Aspiration und andere als zusätzliche Definitionskriterien vorgeschlagen ( Tab.1).

Bei Vorliegen mehrerer ALI-Risikofaktoren soll von einer möglichen TRALI-Reaktion gesprochen werden [18]. Da gerade Patienten mit weiteren Risikofaktoren für ein Lungenversagen besonders gefährdet sind, müssen auch mögliche TRALI-Reaktionen gemeldet werden. Unter den pulmonalen Transfusionsreaktionen ist differenzialdiagnostisch die transfusionsbedingte Herzinsuffizienz infolge Volumenüberladung insbesondere bei bestehenden Herzerkrankungen oder chronischer Niereninsuffizienz [19] („transfusion-associated circulatory overload“, TACO) und der transfusionsassoziierte Asthma-bronchiale-Anfall auszuschließen.

\begin{tabular}{l} 
Tab. 1 Kriterien für eine TRALI-Reaktion (nach [56]). \\
Europäisches Hämovigilanz-Network \\
plötzlich auftretende Atemnot \\
neue bilaterale Lungeninfiltrationen in der Rö-Thoraxaufnahme \\
kein Hinweis für ein kardiogenes Lungenödem \\
Auftreten während oder innerhalb von 6 Stunden nach Bluttransfusion \\
Nordamerikanisches TRALI-Konsensus-Konferenz-Komitee \\
\hline Hypoxämie $\left(\mathrm{PaO}_{2} / \mathrm{FiO}_{2}<300 \mathrm{mmHg}\right.$ oder $\mathrm{O}_{2}$-Sättigung <90\%) \\
keine weiteren Risikofaktoren für ein akutes Lungenversagen (ALI) \\
einschließlich: \\
- Aspiration \\
- Traumata \\
- Pneumonie \\
- kardiopulmonaler Bypass \\
- Brandverletzung \\
- Schadstoffinhalation \\
- Lungentrauma \\
- akute Pankreatitis \\
- Medikamentenintoxikation \\
- Beinahe-Ertrinken \\
- Schock \\
- Sepsis \\
Wenn ein oder mehrere ALI-Risikofaktoren vorliegen, besteht eine mög- \\
liche TRALI.
\end{tabular}

Tab.2 Nachgewiesene Antigene bei TRALI, die mit unterschiedlichen Reaktionen assoziiert sind (nach [56]).

\begin{tabular}{|l|}
\hline Humane leukozytäre Antigene (HLA) \\
\hline HLA Klasse I häufig beteiligt, HLA-A2 bei schweren Reaktionen \\
\hline HLA Klasse Il selten, klinische Bedeutung unklar \\
\hline Humane neutrophile Antigene (HNA) \\
\hline HNA-1a, 1b, 2a selten, aber schwere Reaktionen \\
\hline HNA-3a häufig beteiligt bei lebensbedrohlichen Reaktionen \\
\hline
\end{tabular}

Für Fälle von transfusionsbedingter Atemnot, die sich nicht einer TRALI-Reaktion, einer Herzinsuffizienz oder einem Asthmaanfall zuordnen lassen, empfiehlt die EHN [17] die Bezeichnung „transfusion-associated dyspnea“ (TAD).

\section{Pathophysiologie}

Nach klinischen und experimentellen Studien besteht ein Zusammenhang zwischen dem Vorkommen von leukozytenreaktivierten Antikörpern beim Spender und dem Auftreten von TRALI beim Transfusionsempfänger. Hierbei sind Antikörper im Spenderplasma gegen humane leukozytäre Antigene (HLA) und humane neutrophile Antigene (HNA) und Merkmale der Empfängergranulozyten nachweisbar, die mit einem unterschiedlichen Schweregrad von TRALI assoziiert sind ( $\bullet$ Tab.2). Durch Transfusion von plasmareichen Präparaten werden diese Antikörper auf den Empfänger übertragen und führen zur Bindung an Antigenen auf den Granulozyten des Empfängers, soweit die Granulozyten die entsprechenden Antigene exprimieren. Dabei handelt es sich um eine sogenannte immunogene TRALI [3,20-22]. Auch ist die Beteiligung von Monozyten bekannt [23-25]. Die Granulozyten werden in den Kapillaren der Lungenstrombahn zurückgehalten und setzen Sauerstoffradikale und Enzyme frei, die die Endothelzellen schädigen, sodass Flüssigkeit in die Alveolen austritt und damit ein Lungenödem entsteht, wie es auch bei der ALI oder dem ARDS vorkommt. 
Allerdings wurden nicht in allen TRALI-Fällen leukozytäre oder neutrophile Antikörper nachgewiesen $[3,26]$. Tierexperimentell wurde gezeigt, dass Lipide ebenfalls TRALI in Abwesenheit von Antikörpern auslösen können (nicht immunogene TRALI) [27, 28]. Diese Lipide entstammen sehr wahrscheinlich von Zellmembranen, die während der Lagerung zerstört werden [29]. In Fallstudien wurde dies aber bisher nicht nachgewiesen [30]. Bei 2 Patienten, die an TRALI verstorben sind, wurden in den Lungengefäßen Cholesterinkristalle gefunden [31].

Eine weitere wichtige Beobachtung ist, dass nicht alle Empfänger, die ein Blutprodukt mit leukozytären oder neutrophilen Antikörpern erhalten, auch eine TRALI entwickeln. Diese Beobachtungen führten zu der Hypothese, dass beim Transfusionsempfänger bestimmte immunologische Voraussetzungen vorliegen müssen, bevor eine TRALI eintreten kann [32]. Auf diese Multi-Event-Hypothese weisen Tierexperimente hin, bei denen nur dann eine von Antikörpern induzierte TRALI entsteht, wenn bereits eine Vorschädigung beim Empfänger vorliegt [33]. Dafür sprechen auch die Ergebnisse von Fall-Kontroll-Studien in Verbindung mit TRALI-Risikofaktoren [26].

\section{Risikofaktoren}

Das Risiko für eine TRALI-Reaktion ist erhöht, wenn Blut oder Plasma mit leukozytären Antikörpern der Klasse II und neutrophilen Antikörpern übertragen wird [26]. Mehrfachschwangerschaften können leukozytäre Antikörper produzieren [34], wobei die leukozytären Antikörper der Klasse II gefährlicher als Antikörper der Klasse I sind [26,35].

Höhere Konzentrationen von Interleukin-8 gelten als Marker einer Entzündung. Eine akute Entzündung könnte die erste schädigende Reaktion sein [28], wie experimentelle Daten zeigen [33, 36]. Dadurch werden HLA Klasse II Antigene produziert, die Makrophagen, Neutrophilen und Endothelzellen präsentiert werden und die Produktion von HLA Klasse II Antikörpern auslösen, wobei bestehende Krankheiten die TRALI-Reaktion verstärken $(\bullet$ Tab.3).

Tab. 3 Bekannte Risikofaktoren beim Transfusionsempfänger zur Ausbildung einer TRALI (nach $[26,57]$ ).

\begin{tabular}{l}
\hline hohe Interleukin-8 Konzentration \\
\hline Schock \\
\hline Leberchirurgie \\
\hline chronischer Alkoholabusus \\
\hline positive Flüssigkeitsbilanz \\
\hline $\begin{array}{l}\text { Beatmungssdruck über } 30 \mathrm{~cm} \mathrm{H}_{2} \mathrm{O} \text {, wenn vor der Transfusion beatmet } \\
\text { wurde }\end{array}$ \\
\hline Raucher \\
\hline
\end{tabular}

\section{Klinik (0 Abb. 1)}

Eine TRALI macht sich bemerkbar, indem klinisch zunächst eine ALI innerhalb von 6 Stunden nach Transfusion und in Abwesenheit anderer Risikofaktoren entsteht. TRALI kann bereits in der ersten Stunde nach Transfusion auftreten. Bei beatmeten Patienten weist eine Verschlechterung mit reichlichem bronchoalveolärem Sekret ohne erklärbare Ursachen auf ein TRALI hin. Röntgenologisch sind bilaterale Infiltrate nachweisbar [37]. Fieber und Hypotonie können weitere unspezifische Krankheitszeichen sein.

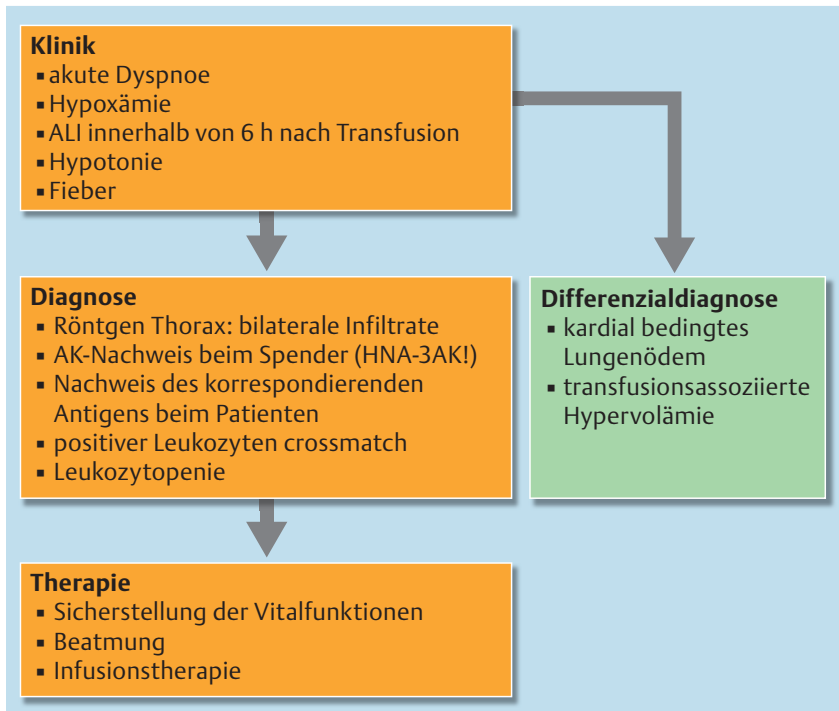

Abb. 1 Algorithmus bei transfussionsassoziierter akuter Lungeninsuffizienz (TRALI).

Tab. 4 Untersuchungen bei TRALI (nach [57]).

Echokardiografie

Brain natriuretic Peptide (BNP)

Proteinanalyse der Sekretflüssigkeit

Weißes Blutbild (Leukozytopenie)

Differenzialdiagnostisch müssen ein kardial bedingtes Lungenödem, transfusionsbedingte Hypervolämie oder andere Ursachen einer akuten Lungeninsuffizienz ausgeschlossen werden. Echokardiografie, Messung der BNP-Konzentration und Analyse von bronchoalveolärer Flüssigkeit sind ergänzende Tests, um kardiale Dysfunktion und Volumenüberladung auszuschließen ( Tab.4). Das Verhältnis der Konzentrationen von bronchoalveolärem Protein zu Plasmaprotein liegt in der Regel über 0,75 [38]. Ein Rechtsherzkatheter ist nicht gerechtfertigt. Eine vorübergehende Leukopenie bei Beginn kann auf eine TRALI hinweisen [39].

Der Nachweis von Antikörpern unterstützt die Diagnose einer TRALI. Antikörper gegen HNA-3 verursachen besonders schwere Formen von TRALI. Da TRALI auch ohne Nachweis von leukozytären Antikörpern auftreten kann, wird die Diagnose eher durch die klinische Symptomatik gestellt. Der Blutbank sollten alle Fälle von Verdacht auf TRALI gem. §63c AMG mitgeteilt werden [40], sodass andere Blutkomponenten des Spenders unter Quarantäne gestellt werden können.

\section{Laboruntersuchung}

Zur Abklärung einer Transfusionsreaktion sollten Spender der beteiligten Blutprodukte, die in einem Zeitraum von bis zu 6 Stunden vor Beginn der Reaktion transfundiert wurden, auf HLA- und HNA-Antikörper untersucht werden [13]. Wird ein leukozytenreaktiver Antikörper identifiziert, sollte auf den Zellen des Empfängers das korrespondierende Antigen nachgewiesen werden. Ein positiver Leukozyten crossmatch sichert die Diagnose. 


\section{Therapie}

$\nabla$

Wie bei der ALI oder dem ARDS, gibt es keine spezifische Behandlung der TRALI. In den meisten Fällen hat TRALI eine bessere Prognose als andere Ursachen, die eine ALI oder ein ARDS auslösen. Der sofortige Beginn einer supportiven Therapie, die in der Regel aus den gleichen Maßnahmen besteht wie bei Patienten mit ALI oder ARDS, verbessert die Prognose erheblich [30]. Bei leichten TRALI-Reaktionen kann die nasale Sauerstoffgabe ausreichen. Für schwere Fälle sind mechanische Beatmung und invasive hämodynamische Überwachung erforderlich. Patienten mit TRALI benötigen hypotone intravenöse Flüssigkeiten, um einen angemessenen Blutdruck aufrechtzuerhalten. Diuretika sind zu vermeiden. In seltenen Fällen kann eine extrakorporale Membranoxygenierung (ECMO) notwendig sein [41,42]. Für Glukokortikoide gibt es keine eindeutige Indikation. Tierexperimentell konnte auch keine Verbesserung durch Methylprednisolon gezeigt werden [43].

\section{Prävention \\ $\nabla$}

Antikörperbedingte TRALI-Reaktionen treten hauptsächlich nach Transfusion von frisch gefrorenem Plasma auf, das von Frauen gewonnen wurde, die sich im Verlauf mehrerer Schwangerschaften gegen leukozytäre Antigene immunisiert haben [18,32,44,45]. Insbesondere multipare Spenderinnen, bei denen Thrombozytound Plasmapheresen durchgeführt worden sind, sollten zur Verringerung des TRALI-Risikos auf leukozytäre Antikörper untersucht werden $[26,46]$. In Deutschland dürfen Blutprodukte von weiblichen Spendern mit Schwangerschaftsanamnese nur dann verwendet werden, wenn die Testung auf humane leukozytäre Antigene der HLA-Klassen I und II sowie auf die humanen neutrophilen Antigene von HNA-1a, HNA-1b, HNA-2a und HNA-3a negativ sind. Diese Testung ist bei einer erneuten Schwangerschaft zu wiederholen.

Die Inzidenz von TRALI ist rückläufig, seitdem vermehrt Plasma von männlichen Spendern verwendet wurde [15,47-51]. Untersuchungen des amerikanischen Roten Kreuzes ergaben, dass TRALI bei den Empfängern, die in den Jahren von 2006 bis 2008 Plasma von männlichen Spendern der Blutgruppen A, B und 0 erhielten, um mehr als das Vierfache zurückging. Dies traf allerdings nicht für die Blutgruppe $A B$ zu [52]. In Deutschland nahmen die TRALI-Meldungen 2010 stark ab ( $\bullet$ Tab.5), und seit 2009 wurden keine TRALI-Todesfälle mehr gemeldet [53,54]. Durch virus-inaktiviertes Plasma (solvent-detergent) wurde bislang kein TRALI verursacht.

Tab.5 TRALI-Meldehäufigkeit pro Millionen transfundierter Einheiten bezogen auf den jeweiligen Zeitraum (nach [58]).

\begin{tabular}{|lcll|}
\hline Produkt & $\mathbf{2 0 0 5 - 2 0 0 8}$ & $\mathbf{2 0 0 9}$ & $\mathbf{2 0 1 0}$ \\
\hline Erythrozytenkonzentrat & 0,46 & 0,88 & 0,22 \\
\hline Thrombozytenkonzentrat & 1,8 & 6,77 & 0 \\
\hline gefrorenes Frischplasma & 11,18 & 4,56 & 0 \\
\hline
\end{tabular}

Bei Thrombozytenkonzentraten kann durch die Anwendung von plasmareduzierten Produkten das Auftreten von TRALI minimiert werden. Durch Entfernung eines Teils des Spenderplasmas unter Zugabe einer Thrombozytenadditivlösung wird antikörperhaltiges Plasma reduziert, sodass die Sicherheit der Hämotherapie optimiert wird [55].

\section{Interessenkonflikt}

$\nabla$

Die Autoren geben an, dass kein Interessenkonflikt besteht.

\section{Literatur}

1 Lanman JT, Bierman HR, Byron RL. Transfusion of leukemic leukocytes in man: hematologic and physiologic changes. Blood 1950; 5: 1099 1113

2 Barnard RD. Indiscriminate transfusion: a critique of case reports illustrating hypersensitivity reactions. NY State J Med 1951; 51: 2399 2402

3 Popovsky MA, Moore SB. Diagnostic and pathogenetic considerations in transfusion-related acute lung injury. Transfusion 1985; 25: $573-577$

4 Gauvin F, Lacroix J, Robillard P et al. Acute transfusion reactions in the pediatric intensive care unit. Transfusion 2006; 46: 1899-1908

5 Rashid N, Al-Sufayan F, Seshia MMK et al. Post transfusion lung injury in the neonatal population. J Perinatol 2013; 33: 292-296

6 Kelly AM, Williamson LM. Neonatal transfusion. Early Hum Dev 2013; 89: $855-860$

7 Lieberman L, Petraszko T, Yi QL et al. Transfusion-related lung injury in children: a case series and review of the literature. Transfusion 2014; 54: $57-64$

8 Gauvin F, Robillard P, Hume H et al. Transfusion-related acute lung injury in the Canadian paediatric population. Paediatr Child Health 2012; 17: $235-239$

9 Holness L, Knippen MA, Simmons $L$ et al. Fatalities caused by TRALI. Transfus Med Rev 2004; 18: $184-188$

10 Kopko PM, Marshall CS, Mackenzie MR et al. Transfusion-related acute lung injury: report of a clinical look-back investigation. JAMA 2002; 287: $1968-1971$

11 Toy P, Popovsky MA, Abraham E et al. National Heart, Lung and Blood Institute Working Group on TRALI. Transfusion-related acute lung injury: definition and review. Crit Care Med 2005; 33: 721 - 726

12 Jaworski K, Maślanka K, Kosior DA. Transfusion-related acute lung injury: A dangerous and underdiagnosed noncardiogenic pulmonary edema. Cardiol J 2013; 20: 337 - 344

13 Serious Hazards of Transfusion (SHOT). Additional cumulative data 1969-2003 und Shot Report 2004. www.shotuk.org

14 Chapman CE, Stainsby D, Jones $H$ et al. Serious Hazards of Transfusion Steering Group. Ten years of hemovigilance reports of transfusionrelated acute lung injury in the United Kingdom and the impact of preferential use of male donor plasma. Transfusion 2009; 49: 440-452

15 Nakazawa $H$, Ohnishi $H$, Okasaki $H$ et al. Impact of fresh frozen plasma from male-only donors versus mixed-sex donors on postoperative respiratory function in surgical patients: a prospective case-controlled study. Transfusion 2009; 49: 2434-2441

16 Mitteilungen des Arbeitsktreises Blut des Bundesministeriums für Gesundheit. Maßnahmen zur Vermeidung der transfusionsinduzierten Lungeninsuffizienz (TRALI). Bundesgesundheitsbl 2009; 52: 572

17 European Haemovigilance Network (EHN). Definitions of Adverse Transfusion Events.www.ehn-org.net

18 Nakagawa $M$, Toy P. Acute and transient decrease in neutrophil count in transfusion-related acute lung injury: cases at one hospital. Transfusion 2004; 44: 1689-1694

19 Murphy EL, Kwaan N, Looney MR et al. TRALI Study Group. Risk factors and outcomes in transfusion-associated circulatory overload. Am J Med 2013; 126: 357.e29-38. DOI 10.1016/j.amjmed.2012.08.019 Epub 2013 Jan 26

20 Sachs UJH, Hattar K, Weissmann $N$ et al. Antibody-induced neutrophil activation as a trigger for transfusion-related acute lung injury in an ex vivo rat lung model. Blood 2006; 107: 1217-1219 
21 Looney MR, Su X, Van Ziffle JA et al. Neutrophils and their Fc gamma receptors are essential in a mouse model of transfusion-related acute injury. J Clin Invest 2006; 116: 1615-1623

22 Kanai R, Iijima T, Hashimoto $S$ et al. Impact of immunoreactive substances contained in apheresis platelet concentrate on postoperative respiratory function in surgical patients receiving platelet transfusion: a prospective cohort study. Transfus Med 2013. DOI 10.1111/tme.12056 [Epub ahead of print]

23 Land WG. Transfusion-related acute lung injury: The work of DAMPs. Transfus Med Hemother 2013; 40: 3-13

24 Sachs UJ, Wasel W, Bayat B et al. Mechanism of transfusion-related acute lung injury induced by HLA class II antibodies. Blood 2011; 117 : $669-677$

25 Strait RT, Hicks W, Barasa N et al. MHC class I-specific antibody binding to nonhematopoietic cells drives complement activation to induce transfusion-related acute lung injury in mice. J Exp Med 2011; 208: $2525-2544$

26 Toy P, Gajic O, Bacchetti P et al. TRALI Study Group. Transfusion-related acute lung injury: incidence and risk factors. Blood 2012; 119: 1757 1767

27 Silliman CC, Voelkel NF, Allard JD et al. Plasma and lipids from stored packed red blood cells cause acute lung injury in an animal model. J Clin Invest 1998; 101: 1458-1467

28 Silliman CC, Bjornsen AJ, Wyman TH et al. Plasma and lipids from stored platelets cause acute lung injury in an animal model. Transfusion 2003; 43: 633-640

29 Silliman CC, Clay KL, Thurman GW et al. Partial characterization of lipids that develop during the routine storage of blood and prime the neutrophil NADPH oxidase. J Lab Clin Med 1994; 124: 684-694

30 Vlaar AP, Hofstra JJ, Determann RM et al. The incidence, risk factors, and outcome of transfusion-related acute lung injury in a cohort of cardiac surgery patients: a prospective nested case-control study. Blood 2011; 117: $4218-4225$

31 Van Ness M, Jensen H, Adamson GN et al. Neutrophils contain cholesterol crystals in transfusion-related acute lung injury (TRALI). Am J Clin Pathol 2013; 140: 170 - 176

32 Silliman CC, Paterson AJ, Dickey WO et al. The association of biologically active lipids with the development of transfusion-related acute lung injury: a retrospective study. Transfusion 1997; 37: 719-726

33 Looney MR, Nguyen JX, Hu Y et al. Platelet depletion and aspirin treatment protect mice in a two-event model of transfusion-related acute lung injury. J Clin Invest 2009; 119: 3450 - 3461

34 Triulzi DJ, Kleinman S, Kakaiya RM et al. The effect of previous pregnancy and transfusion on HLA alloimmunization in blood donors: implications for a transfusion-related acute lung injury (TRALI) risk reduction strategy. Transfusion 2009; 49: 1825-1835

35 Reil A, Keller-Stanislawski B, Günay S et al. Specificities of leucocyte alloantibodies in transfusion-related acute lung injury and results of leucocyte antibody screening of blood donors. Vox Sang 2008; 95: $313-317$

36 Kelher MR, Masuno T, Moore EE et al. Plasma from stored packed red blood cells and MHC class I antibodies causes acute lung injury in a 2event in vivo rat model. Blood 2009; 113: 2079-2087

37 Carcano C, Okafor N, Martinez F et al. Radiographic manifestations of transfusion-related acute lung injury. Clin Imaging 2013; 37: 10201023

38 Yost CS, Matthay MA, Gropper MA. Etiology of acute pulmonary edema during liver transplantation : a series of cases with analysis of the edema fluid. Chest 2001; 119: 219-223
39 Looney MR, Gropper MA, Matthay MA. Transfusion-related acute lung injury: a review. Chest 2004; 126: $249-258$

40 Gesetz über den Verkehr mit Arzneimitteln (AMG).

41 Lee AJ, Koyyalamudi PL, Martinez-Ruiz R. Severe transfusion-related acute lung injury managed with extracorporeal membrane oxygenation (ECMO) in an obstetric patient. J Clin Anesth 2008; 20: 549-552

42 Kuroda H, Masuda Y, Imaizumi H et al. Successful extracorporeal membranous oxygenation for a patient with life-threatening transfusionrelated acute lung injury. J Anesth 2009; 23: 424-426

43 Müller MC, Tuinman PR, van der Sluijs KF et al. Methylprednisolone fails to attenuate lung injury in a mouse model of transfusion related acute lung injury. Transfusion 2013. DOI 10.1111/trf.12394 [Epub ahead of print]

44 Gajic O, Rana R, Mendez JL et al. Acute lung injury after blood transfusion in mechanically ventilated patients. Transfusion 2004; 44: 1468 1474

45 Brander L, Reil A, Bux J et al. Severe transfusion-related acute lung injury. Anesth Analg 2005; 101: 499-501

46 Carrick DM, Norris PJ, Endres RO et al. National Heart, Lung, and Blood Institute Retrovirus Epidemiology Donor Study-II. Establishing assay cutoffs for HLA antibody screening of apheresis donors. Transfusion 2011; 51: $2092-2101$

47 SHOT. SHOT Annual Reports and Summaries (All). 2010. http://www shotuk.org/wp-content/uploads/2011/07/SHOT-2010-Report1.pdf

48 Fatalities reported to FDA following blood collection and transfusion: Annual summary for fiscal year 2011. 2011: http://www.fda.gov/BiologicsBloodVaccines/SafetyAvailability/ReportaProblem/TransfusionDonationFatalities.htm

49 Eder AF, Herron RM Jr, Strupp A et al. Effective reduction of transfusionrelated acute lung injury risk with male-predominant plasma strategy in the American Red Cross (2006-2008). Transfusion 2010; 50: 1732 1742

50 Transfusion-Related Acute Lung Injury. AABB Association Bulletin 2006. http://www.aabb.org/programs/publications/bulletins/Pages/ ab06-07.aspx

51 Clarifications to recommendations to reduce the risk of TRALI. AABB Association Bulletin 2007.http://www.aabb.org/programs/publications/bulletins/Pages/ab07-03.aspx

52 Eder AF, Dy BA, Perez JM et al. The residual risk of transfusion-related acute lung injury at the American Red Cross (2008-2011): limitations of a predominantly male-donor plasma mitigation strategy. Transfusion 2013; 53: $1442-1449$

53 Tank S, Sputtek A, Kiefmann R. Transfusionsassoziierte akute Lungeninsuffizienz. Anaesthesist 2013; 62: 254-260

54 Funk MB, Guenay S, Lohmann A et al. Benefit of transfusion-related acute lung injury risk-minimization measures - German haemovigilance data (2006-2010). Vox Sanguinis 2012; 102: 317-323. DOI 10.1111/j.1423-0410.2011.01556.x

55 Van der Meer PF, Kerhoffs JL, Curvers J et al. In vitro comparison of platelet storage in plasma and in four platelet additive solutions and the effect of pathogen reduction: a proposal for an in vitro rating system. Vox Sang 2010; 98: $517-524$

56 Reil A, Bux J. Transfusionassoziierte akute Lungeninsuffizienz. Dtsch Ärztebl 2007; 104: A 1018-1023

57 Sayah DM, Looney MR, Toy P. Transfusion reactions: newer concepts on the pathophysiology, incidence, treatment, and prevention of transfusion related acute lung injury (TRALI). Crit Care Clin 2012; 28 : $363-372$

58 Funk M, Günay S, Volz-Zang C. Hämovigilanz-Bericht des Paul-EhrlichInstituts. 2010 